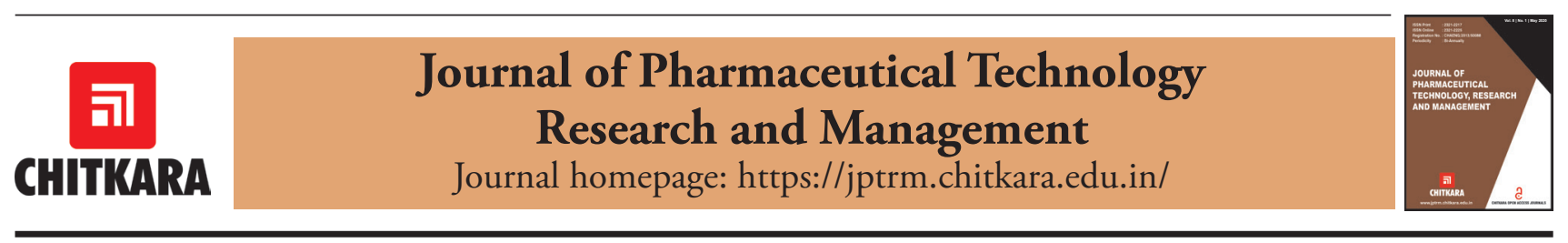

\title{
Pharmaceutical Applications of Xanthan Gum in Ophthalmic Delivery Systems
}

\author{
Shiveena Bhatia ${ }^{1}$, Tarun Kumar $^{2}$, Sonali Batra ${ }^{3}$ and Sumit Sharma ${ }^{4 *}$ \\ ${ }^{1,2,4}$ Chitkara College of Pharmacy, Chitkara University, Rajpura, Punjab-140401, India \\ ${ }^{3}$ Deparment of Pharmaceutical Sciences, Guru Jambheshwar University of Science and Technology, Hisar, Haryana-125001, India \\ ${ }^{1}$ shiveena18084.ccp@chitkara.edu.in \\ ${ }^{2}$ tarun19509.ccp@chitkara.edu.in \\ ${ }^{3}$ sonalibatra88@gmail.com \\ 4*sumit.sharma@chitkara.edu.in (Corresponding Author)
}

\section{ARTICLE INFORMATION}

Received: December 24, 2019

Revised: February 21, 2020

Accepted: March 27, 2020

Published Online: May 20, 2020

Keywords:

Xanthan gum, Viscosifier, Mucoadhesive,

Chemical modification, Biodegradable

\section{ABSTRACT}

Introduction: Ophthalmic delivery system is one of the challenging domains of formulation and development due to tear dilutions, drug loss due to lacrimal drainage, limited volume and pre-corneal barriers. Several pharmaceutical technologies are exploited in order to counter the challenges posed by ocular route such as emulsions and suspensions. But all these technologies have stability issues which lead to their limited use.

Background: Among polysaccharides, xanthan gum, a natural occurring biodegradable exopolysaccharide extracted from bacterium Xanthomonas campestris is widely accepted as one of the potential polysaccharide in ophthalmic delivery systems.

Review Results: Xanthan gum is commonly used as an additive to various ophthalmic formulations due to its mucoadhesive property and imparting stability to various novel pharmaceutical technologies for ophthalmic. Xanthan gum also allows chemical modifications with various ligands which consequently allow controlled release, modified dissolution rate and viscoelasticity. Conclusion: In this review we are providing an insight over potential of pharmaceutical applications of xanthan gum. Also, we have discussed the scope of chemical modifications in xanthan gum with modified physicochemical properties.

\section{Introduction}

The increase demand of environmental concerns over non-biodegradable polymers have been driving forward with the development of biodegradable polymers, due to its cost effective availability and biodegradability. Apart from environmental friendly these polymers also possess properties like mucoadhesiveness, film-forming ability and easily tailored to impart stimulus responsive activation. Such properties allow their wide range of application in pharmaceutical industry. Ophthalmic delivery system is one of the challenging domains of formulation and development due to lacrimal drainage, tear dilutions, non productive absorption and relatively less permeable through the epithelial cells of eye (Lallemand, Felt-Baeyens, Besseghir, Behar-Cohen \& Gurny, 2003). To overcome such difficulties in ophthalmics use of certain polysaccharides and their modifications are proven to be a promising approach(Sharma \& Batra, 2019; Sharma, Sinha, Sarwal \& Shukla, 2018; Sumit, Shikha, \& Rayasa, 2012). Xanthan gum, a natural occurring biodegradable exo-polysaccharide extracted from bacterium Xanthomonas campestris is widely accepted as one of the potential polysaccharide in ophthalmic. Xanthan gum is discovered in the late 1960's by the team of United States Food and Drug Administration (USDA) researchers. It is widely used in many important preparations ranges from food, cosmetics, oil extracting industries and pharmaceutical products. Xanthan gum has a broad range of operations that makes it substance of first choice in ophthalmic preparations as thickening agent, suspending agent or as stabilizing agent to stabilize the $\mathrm{pH}$ of the preparation to make it compatible with the $\mathrm{pH}$ of target site (Benita \& Nassar, 2016). The development of an ocular dosage form containing xanthan gum is capable of interacting with mucin in the precorneal area which is a challenge, because limited number of patients has the acceptability in response with the highly viscous preparations. This is due to the low concentration of mucin at precorneal site and high ionic strength lead to organization of xanthan gum structure. This results in limited interaction of polymer with heterotypic junctions. In this review article 
we are discussing the physicochemical properties of xanthan gum in different conditions required for ideal ophthalmic delivery system. Also, the chemical modifications of xanthan gum reported in literature with respect to improvising its practical applications in ophthalmic.

\section{Chemistry of Xanthan Gum}

Xanthan gum is a biodegradable natural polymer obtained from bacterium Xanthomonas campestris on cabbage plants. Xanthan gum is a trisaccharide compound having a large molecular mass. The basic chain present in xanthan gum is $1,4 \beta$-D-Glucose residue. Xanthan gum consists of D-glucosyl, D-glucuronyl and D-mannosylacid residues with molar ratio 2:1:2 (Dário, Hortêncio, Sierakowski, Neto, \& Petri, 2011). The secondary structure of xanthan gum consists of a right-handed, five-fold helix with a rise per backbone disaccharide residue, i.e. five-fold helix. It gives more strength and holds the structure in aligned and proper manner. Production of xanthan gum is raised commercially on global level as emergent excipient for ocular drug delivery in eye. Xanthan gum for their tremendous advantages currently isbeing used as preferred additive for the various ophthalmic preparations for their properties. Along with ocular drug delivery xanthan gum has diverse and wide range of contribution in various edible and cosmetic products. Due to saccharide in nature it has unique advantage to enhance viscosity of the solution; others are lubricant and stabilizing agent. Xanthan gum is soluble in both hard and soft water. Formation of lumps gets started when directly added to water so it is mandatory to pass the xanthan gum from aggregation process to reduce the size of particles and maintain the uniformity of the solution. It attains solution state when placed under elevated temperature. At initial or rest state when no external shear is applied hydrogen bonding holds xanthan gum in its limited boundary. Number of modifications would be done by implicating different chemical processes to increase the rheological characters of xanthan gum such as carboxymethylation, microwave-assisted, chemoenzymatic and plasma assisted chemical grafting to alter physicochemical properties. It also exerts bioadhesive property and become the main advantage over the drawback of limited absorption in ophthalmic preparations. Bioadhesion is tendency of substance to make a strong linkage with biological tissues and prevent loss due to lacrimal drainage. In modern approaches to ocular drug delivery the first priority of any pharmaceutical industry is to ensure the safety of preparations along with their efficacy. In currently applied methods the implication of synthetically derived polymers as additives along with active chemical ingredients is great combination for ophthalmic preparations.

\section{Physiochemical Behaviour of Xanthan Gum}

\subsection{Effect of Temperature}

Temperature is one of the significant parameter which affects the physiochemical properties of the xanthan gum. Change in temperature from optimum range leads to change in conformation from ordered to disordered state. In a report submitted by Brunchi and co-workers revealed changes occurred in the electrokinetics and the flow behavior on the aqueous xanthan gum when dissolved at high temperature. This study reveals that increase in the viscosity of xanthan gum due to reshaping in the conformations of particles from ordered to disordered takes place at concentration range of $0.1-0.3 \mathrm{~g} / \mathrm{dl}$ with elevation in temperature upto $40^{\circ} \mathrm{C}$. The rational statement given by the author for the increase in viscosity with respect to temperature is imbalance between Coulombic and non-Coulombic electrostatic forces of interactions (Brunchi, Avadanei, Bercea, \& Morariu, 2019).

\subsection{Effect of $p H$}

Hydrogels are one of the promising approaches for the better and efficacious results in the ocular drug delivery. Hydrogels formed by crosslinking process where it involves addition of physical or chemical cross linking agents for polymerization of their subunits. Swelling ratio is the characteristic property of hydrogels which is greatly influenced by $\mathrm{pH}$. For instance, in hydrogels based on xanthan gum with increase in $\mathrm{pH}$ particularly above 6.5 , swelling ratio increases. This effect is due to prominent electrostatic repulsions within the gel crosslinked structure. Moreover, deprotonation of unreacted hydroxyl groups and ester bond hydrolysis in alkali medium are also contributes to this effect (Bueno, Bentini, Catalani, $\&$ Petri, 2013).

\subsection{Effect of Concentration}

In ophthalmic preparations, the viscosity of upto $20 \mathrm{mPa}$. S is considered to be optimum and tolerant. It is observed that xanthan gum concentration follows linear relationship with viscosity at low concentrations range upto $1.0 \%$. Also at higher concentration of above $1.0 \%$ the aqueous dispersion of xanthan gum shows gel like consistency at normal state (Zatz \& Knapp, 1984). In a study at a concentration range of $0.2,0.6$ and $1.0 \%$ of xanthan gum at a shear rate of $300 \mathrm{~s}^{-1}$ the viscosity ranges approximately as $9.5,36$ and $120 \mathrm{mPa}$. S respectively. The viscosity less than $20 \mathrm{mPa}$. $\mathrm{S}$ is optimum in case of Newtonian and pseudoplastic systems for ocular delivery. Higher viscosities are not suitable for use in ophthalmic with Newtonian and pseudoplastic systems. In addition to this, the systems show viscoelastic behavior the viscosity of around $150 \mathrm{mPa}$. S are considered optimum for ophthalmic use (Ceulemans, Vinckier \& Ludwig, 2002). 


\section{Pharmaceutical Applications}

\subsection{Xanthan Gum as Mucoadhesive}

Mucoadhesion is the phenomenon which holds two materials together by managing interfacial forces between them to enhance the time of retention of the active substance and prolongs the therapeutic effect. Mucoadhesion is a significant approach which overcomes certain drawbacks of traditional drug delivery such as binding with lachrymal protein, tear turnover, low precorneal penetration and noneffective absorption. Alteration of certain physiochemical factors of polymer such as concentration, molecular conformation, molecular mass imposes significant impact on mucoadhesive behavior of the polymer with mucin. Mucin is a glycosylated protein present in eyes at very low concentrations (Mann, Campbell \& Tighe, 2016).

In a report, submitted by Morsi and co-workers evidenced the use of xanthan gum to increase the mucoadhesive property of in situ nanoemulsion gelling system. In this experimental study, gellan gum was used in combination with certain polymers (xanthan gum, HMPC K4M and carbopol 940) for the preparation of in situ gelling system. For the preparation of aqueous formulation, deionized water was used and polymeric combination (gellan/carbopol 940, gellan/xanthan and gellan/HPMC) were dissolved in it. This process was done with continuous heating for $20 \mathrm{~min}$ at $90^{\circ} \mathrm{C}$. Then acetazolamide loaded nano emulsion was added into the above mentioned aqueous polymeric dispersion. The mucoadhesive strength of finished product comprising variable polymeric concentrations $(0.2$, 0.4 and $0.6 \% \mathrm{w} / \mathrm{w}$ ) with $0.3 \% \mathrm{w} / \mathrm{w}$ fixed concentration of gellan gum was determined. The pattern of mucoadhesive behavior at concentration $0.2 \%$ was carbopol $940>$ xanthan gum > HPMC. Further increase in concentrations results in change in relative mucoadhesive behavior of gellan gum except carbopol. The mucoadhesive behavior of the carbopol was neither increased nor decreased when the strength was increased to $0.4 \%$ and $0.6 \%$. The order of mucoadhesive behavior after increase in strength are xanthan gum > carbopol > HPMC. This study evidenced the mucoadhesive behavior is due to induced ionic charge on the side chain of the polymer. The charged group on the side chains makes its molecule enlarged due to its electrostatic repulsive force. This leads to the formation of hydrogen bonding and makes the molecule align by formation a helical structure which holds water in the molecule and results increase the viscosity of in situ gelling system. Then further increased in concentration enhances the molecular bulk and makes the side chains more interlinked and enhances the viscosity of in situ gelling system as compared to other polymers (Morsi, Ibrahim, Refai \& El Sorogy, 2017).
In another study conducted by Ahmedand co-workers evidence use xanthan gum in combination with hydroxyl propyl methyl cellulose (HPMC E50LV) to enhance the mucoadhesion and release time of in situ gel preparation of brimonidine tartrate. Concentration of xanthan gum used in the experiment was $(0.21 \%)$. For the preparation of in situ gels, borate buffer solution was taken and heated up to $70^{\circ} \mathrm{C}$ after the inclusion of sufficient quantity of polymer. Addition of drug was followed after the addition of required quantity of xanthan gum with slight heating and mixed completely in the solution. In the above prepared solution phenylethyl alcohol and HPMC E50 LV were added by giving the exposure of magnetic stirring. Then final product was sterilized by applying autoclaving technique. Experiment was performed on the basis of $2^{3}$ factorial design protocol. After the completion of whole study it was evidenced that xanthan gum at optimum concentration $(0.21 \%)$ does not have any interaction with the drug and increase in sustained release of drug was observed up to 10 hours (Ahmed \& Goli, 2018).

\subsection{Xanthan Gum as a Stabilizer}

Ocular drug delivery demands amphiphilic characteristics to get absorbed quickly for good bioavailability and tissue distribution. As the barriers in ophthalmic is the aqueous layer of lacrimal glands followed by lipid layer of meibomian glands. Drugs which are highly hydrophilic or lipophilic in nature exert insufficient bioavailability and tissue distribution through ocular route. Therefore, emulsions are one of the promising drug delivery systems to meet the prime requisites for efficient ocular drug delivery. But emulsions have stability issues due to coalescence which ultimately lead to phase separation. Moreover, suspensions are also commonly exploited for ophthalmic formulations. This delivery system has a stability issue due to cake formations which ultimately lead to phase separation. Natural polymers like xanthan gum for their magnificent effect to modify the rheological characters of pharmaceutical formulations by resulting the change in shear thinning property and change in movement kinetics when used in optimum concentration range are commonly used in ophthalmic.

There are certain problems which make the drug less bioavailable such as creaming during storage time, gravitational separation, coalescence, flocculation, phase inversion and thermostability. Xanthan gum in a variety of formulations such as emulsions, in situ gelling with certain modifications are used as stabilizers to enhance the bioavailability and stability of ophthalmic formulations. Xanthan gum with certain chemical modifications is proven to minimize the interfacial tension by acquiring amphiphilic characteristics and enhance the viscosity of continuous 
phase. This reduces the particle movement in between the system and prevents coalescence as well as settling of colloidal particles.

In a report, submitted by Céline and co-workers hydrophobically modified xanthan gum of optimum concentration range was taken to prepare oil-in-water emulsion. Grafting of polysaccharide was done with octyl residue on its carboxylic group through peptide coupling reaction. Hydrophilic solutions of hydrophobically modified xanthan with concentration ranges from $0.125 \%$ to $1.125 \% \mathrm{w} / \mathrm{w}$ were prepared with continuous overnight stirring at 20 to $25^{\circ} \mathrm{C}$. The author concluded that at low concentration of modified xanthan gum i.e. from 0.05 to $0.1 \% \mathrm{w} / \mathrm{w}$ emulsion was observed to be unstable. A phase separation with non uniform oil droplet size was observed rendering the emulsion as unstable on storage. Furthermore, on increasing the concentration of modified xanthan gum uptill $0.2 \% \mathrm{w} / \mathrm{w}$ a homogenous emulsion with uniform droplet size was observed (Céline et al., 2018).

In another study, submitted by Krstonošić and coworkers increased concentration of xanthan gum $0.01 \%$, $0.08 \%$ up to $0.12 \%$ was used to overcome the creaming problem in emulsion. At initial concentration of xanthan gum $0.01 \% \mathrm{w} / \mathrm{w}$ lipophilic droplets phase got flocculated and clear separated biphasic system is formed. Aggregation of oil droplets was observed at the top as well as at the bottom of the aqueous continuous system. The strength of interconnected network of droplets was decreased when concentration of gum was increased from $0.04 \%$ to $0.08 \%$ $\mathrm{w} / \mathrm{w}$ and got reunited due to certain factors such as Brownian motion, gravitational force. Further increased concentration of xanthan gum up to $0.12 \%$ leads to the emergence of critical viscosity stage and results in the intellectual disability of creaming by deceleration the establishment of network of droplets. In addition to this, xanthan gum enhanced the aqueous phase stability of the emulsion by creating the uniformity in the size distribution of droplets with enhanced interfacial surface area and also decrease the flow kinetics of the emulsion (Krstonošić et al., 2009).

\subsection{Xanthan Gum as Viscosifier}

In current days the approaches and demands of the ocular treatments have become more advanced targeting to the required site. Nano particle drug delivery is promising approach in various dosage forms (suspensions, emulsions and ointments) to produce optimum effects. In ocular delivery leading barriers which restrict the drug for its action, are less corneal retaining time, rapid eye blinking, blood retinal permeation and low penetrating tendency. To maintain the viscosity in optimum range of the formulation in ocular delivery and to achieve the optimum effect of prepared formulation a range of $15-150 \mathrm{mPa} s$ has been defined. In this optimum viscosity range penetration of drug from corneal layer is maximum and there is decreased frequency of administration of the drug. Sudden increase in viscosity from the optimum range exerts negative effect such as blurring of vision and increase the residence time from the required time range in deeper tissues of the eye.

In a report submitted by Ceulemans et al. study the effect of viscosity when xanthan gum of optimum concentration $(1 \% \mathrm{w} / \mathrm{v})$ was mixed with mucin ranging between $8 \%$ and $16 \%$ for the optimum rheological characteristics. When the mixture got cooled after the heating, changes took place and the conformation changed from ordered to disordered state and the whole sample could not attain its initial helix structure. This resulted in increase in formation of secondary bond or physical entanglement between mucin and xanthan gum as a result increasing its intrinsic viscosity (Ceulemans, Vinckier \& Ludwig, 2002).

\subsection{Xanthan Gum as Supportive Agent for Synthetic Polymers}

Instant drainage and secretion of lachrymal fluid are the major problems associated with ophthalmic delivery. Synthetic polymers like poloxamer are observed to be promising material with stimuli responsive properties against temperature to overcome from aforesaid physiological considerations. At specific concentration the aqueous dispersion of these polymers shows reversible sol to gel transformations under the influence of temperature. One of the limitations of poloxamer gel is their low mucoadhesive property. To overcome this type of disadvantages xanthan gum is used as supportive agent. Bhowmik and co-workers submitted a report to show the effect of xanthan gum used in combination with guar gum in optimum ratio on in situ gelling poloxamer system. This study revealed the formation of poloxamer gel when temperature was increased up to physiological temperature $37^{\circ} \mathrm{C}$ in an optimum concentration range of $18-20 \%$. When concentration decreased from the optimum concentration there was no formation of gel. The study revealed that the combination of xanthan gum and guar gum in ratio of 3:7 induced the formation of gel (Bhowmik et al., 2013).

\section{Chemical Modification of Xanthan Gum}

As we discussed above that xanthan gum is used for diverse applications such as stabilizing agents, thickening agent, bioavailability enhancer and as a commercial pharmaceutical aid, there are some limitations associated with it. Some barriers such as slow dissolution rate, low molecular strength, aggregates the gum and leads to the 
formation of the lumps and delays the optimum therapeutic index in the required time. So, to overcome these barriers, certain chemical modifications such as carboxymethylation, grafting, copolymerization of xanthan gum is done (Ahuja, Kumar \& Singh, 2012).

\subsection{Cold Plasma Treatment}

Cold plasma treatment is one of the techniques explored for chemical modifications of xanthan gum. It is a non thermal technique which is used to inactivate the microbial action that is due to the exposure of xanthan with free radicals, electric field, UV light and other chemically excited species. It affects the physiological and thermal properties of xanthan gum. It also shows grateful impact on secondary and tertiary structure of the xanthan gum. At the same time it has no effect on the nutrition composition of xanthan which includes moisture, crude ash, and nitrogen free extracts. Outcomes of cold plasma treatment is decrease in bulk, tap density and compressibility index with an increase in porosity, Hausner's ratio and angle of repose of xanthan gum. Other results of cold plasma treatment are change in acid, hydroxyl value and $\mathrm{pH}$ of xanthan gum.

\subsection{Formaldehyde as a Dissolution Enhancer}

For the better efficacy and optimum xanthum gum therapeutic response more dispersibility of excipient in the formulation is one of the essential demanding approaches. One of the limiting properties of xanthan gum is its slow dissolution rate.

In a study performed by Suand co-workers, effect of formaldehyde on the dissolution rate of xanthan gum was examined. The study showed that purified modified gum had rapid dissolution rate over xanthan gum alone. The optimum increase in the viscosity was observed in initial time range $0-4$ seconds. The sudden increase in the swelling and dissolution due to change in molecular conformation with addition of formaldehyde was observed. This was attributed to limit the intermolecular bonding and ordered-disordered state in polymer (Badwaik et al., 2013; Su et al., 2003).

\subsection{Etherification of Xanthan Gum}

For the optimum therapeutic and rheological responses in ocular drug delivery modification in the basic structure of xanthan gum is one of the promising approach. Xanthan gum when dissolved in aqueous medium exerts limited swelling capacity due to the formation of gelatinous layer which results in the slow hydration of polymer and restricts the rate of dissolution. Carboxymethylation is one of the currently used techniques for the modification of the xanthan gum in order to improve polymer dissolution and mucoadhesion. Carboxymethylation is done by attachment of monochloracetic acid by substituting hydroxyl group attached on the main chain of the xanthan gum. This substitution of hydroxyl group is known as etherification and is basically the process of dehydration of hydroxyl group when polysaccharide reacts with carboxymethyl group. For the customization of the release profile and to enhance the mucoadhessive property of the xanthan gum, carboxymethylation is done (Hamcerencu, Popa, Riess \& Desbrieres, 2019).

In another report submitted by Ahuja and co-workers, carboxymethylation of xanthan gum was done by reacting monochloroacetic acid with xanthan gum. This study revealed that carboxymethylated xanthan gum resulted in minimizing the size of particles as compared to pure xanthan gum and change in the shape of particle from irregular to spherical. Spherical particles have certain advantages over irregular particles as it enhances the flow property of the ophthalmic preparations, increases the rate of hydration and rapid dissolution of xanthan gum (Ahuja et al., 2012).

\subsection{Grafting of Xanthan Gum}

Grafting is the process where different blocks are connected to the main chain of the host polymer as side chains. All these blocks have different characteristic properties as compare to the main chain character. Grafting reaction is based on copolymerization of two or more replicating monomeric units The principle based on grafting polymerization is the cohort of free radicals on the host polymer, and promote polymerization of monomers into free radical sites resulting into graft copolymerization. These copolymers are differentiated as random copolymers, block copolymers, alternate copolymers and graft copolymers. Grafting technique is used to modify the features of the host polymers such as solubility, permeability capacity, reactivity, elasticity and temperature. In recent centuries, grafting copolymerization technique has expanded based on the modification of natural polysaccharides. Different types of grafting techniques include - chemical method, microwave radiation- induced grafting, enzymatic grafting and plasmainitiated grafting. Chemical method involves chemical redox initiators (Lewis acids, metal carbonyl, strong bases etc.) to produce free radicals or active sites on the host polymer. Microwave radiation - induced grafting is most convenient form of grafting. It involves active sites or free radicals when electromagnetic radiation is passed through the host polymer. This technique maintains the highest purity, subsequently prevents contamination, toxic solvents, reduces the time of reaction and provides high yields. In enzymatic grafting, an enzyme induces a chemical grafting on the host polymer. The grafting of natural polysaccharides with the help of enzyme has been used in large number of 
industries. Certain organic chemicals used for the grafting of xanthan gum are maleic anhydride, acrylic acid and acryloyl chloride (Hamcerencu et al., 2019; Kulkarni et al., 2019).

\subsection{Grafting with Acrylamide}

Modification of the xanthan gum through hydrolysis and grafting it with acrylamide is usually done to improve its functional characteristics with enhancing range of applicability. For instance, poly (N-isopropyl acrylamide) based hydrogel has been extensively studied as an intelligent polymeric matrix for use in biotechnology and bioengineering. It is also investigated as biocompatible for human use.

Badwaik and co-workers prepared the Poly amalgamate of acrylamide-grafted-carboxymethyl xanthan gum by liquefying dry carboxymethylated xanthan gum of concentration $4-24 \mathrm{~g} / \mathrm{dm}^{-3}$ in distilled water. The study revealed the enhancement in the rheological characteristics of the blend due to increase in the number of active site when the concentration of the carboxymethylated xanthan gum was taken in the optimum range of $4-16 \mathrm{~g} / \mathrm{dm}^{-3}$. Further increase in the concentration made the blend more viscous and retarded the motion of particles. Study concluded that the intrinsic viscosity of the carboxymethylated xanthan gum was decreased due to the formation of polyacrylamide at hydroxyl group. This in turn, decreased the intrinsic viscosity and hence the flow becomes non-Newtonian. Study also evidenced the increase in the thermal stability of the polyacrylamide carboxymethylated xanthan gum as compared to carboxymethylated xanthan gum with reduction in semi crystalline nature of particles due to hindrance in the ordered structure (Badwaik et al., 2016).

\subsection{Grafting with acryl Acid}

Multiple acrylic acid units have been prepared and investigated for their ability to act as controlled release vehicles in ophthalmic drug delivery especially delivering to corneas. In sustainable drug delivery, thermoresponsive in situ gels have been utilized to improve ocular absorption because of their lower critical solution temperature (Rosalam \& England, 2006). Poly-(N-isopropyl acrylamide) (PNIPAAm)-based thermoresponsive copolymers have been extensively studied owing to the thermally induced phase transition of PNIPAAm at $32^{\circ} \mathrm{C}$, which is close to the physiological temperature.

The study conducted by Hajikhani and co-workers evidenced the use of $\mathrm{N}, \mathrm{N}^{\prime}$-hexane-1, 6-dilbisprop-2enamide or 1, 4-butandioldimethacrylate as cross-linking agent which assisted the formation of semi- interpenetrating polymer network by entrapping the polysaccharide inside the crosslink agent and exert their action on acrylic acid/ xanthan gum biopolymer complex. The study evidently confirms the increase in stability of xanthan gum in different phase. Formation of inter penetrating networks was found to be the reason for improved mechanical strength and ultimately contributing to the stability of formulation (Hamcerencu et al., 2019; Kulkarni et al., 2019).

\section{Conclusion}

Many attempts have been made to improvise the ophthalmic formulations with respect to increasing bioavailability and imparting stability to the emulsions and suspensions for longer shelf life. Xanthan gum provides numerous opportunities because of multiple functionalities. Providing with different possibilities of conjugation with numerous ligands and structural modifications has led xanthan gum as potential biopolymer for ophthalmic applications. Several detailed evidence based studies have been done on xanthan gum describing the characteristic behavior of xanthan gum in various physiological conditions. In our opinion, xanthan gum is an ideal biopolymer for controlled release, stabilizer for emulsions and suspensions, as dissolution enhancer and improving bioavailability for ophthalmic preparations.

\section{Authorship Contribution}

Dr Sumit Sharma and Dr Sonali Batra, the principal investigators of the present review contributed in designing of the manuscript, the supervision of the work done and undertook a critical reading of the same. Miss Shiveena Bhatia and MrTarun Kumar contributed in rigrous literature survey and collection of apt data and drafting the manuscript.

\section{Conflict of Interest}

Authors have no conflict of interest.

\section{References}

Ahmed, V.A., \& Goli, D. (2018). Development and Characterization of In Situ Gel of Xanthan Gum for Ophthalmic Formulation Containing Brimonidine Tartrate. Asian Journal of Pharmaceutical and Clinical Research, 11(7), 277-284. https://doi.org/10.22159/ajpcr.2018.v11i7.25221

Ahuja, M., Kumar, A., \& Singh, K. (2012). Synthesis, characterization and in vitro release behavior of carboxymethyl xanthan. International Journal of Biological Macromolecules, 51(5), 1086-1090.

https://doi.org/10.1016/j.ijbiomac.2012.08.023 
Badwaik, H.R., Giri, T.K., Nakhate, K.T., Kashyap, P., \& Tripathi, D. K. (2013). Xanthan gum and its derivatives as a potential bio-polymeric carrier for drug delivery system. Current Drug Delivery, 10(5), 587-600. https://doi.org/10.2174/1567201811310050010

Badwaik, H.R., Sakure, K., Alexander, A., Ajazuddin, Dhongade, A.H., \& Tripathi, D.K. (2016). Synthesis and characterisation of poly(acryalamide) grafted carboxymethyl xanthan gum copolymer. International Journal of Biological Macromolecules, 85, 361-369. https://doi.org/10.1016/j.ijbiomac.2016.01.014

Bhowmik, M., Kumari, P., Sarkar, G., Bain, M., Bhowmick, B., Mollick, M., . . . Chattopadhyay, D. (2013). Effect of xanthan gum and guar gum on in situ gelling ophthalmic drug delivery system based on poloxamer-407. International Journal of Biological Macromolecules, 62, 117-123.

https://doi.org/10.1016/j.ijbiomac.2013.08.024

Brunchi, C.-E., Avadanei, M., Bercea, M., \& Morariu, S. (2019). Chain conformation of xanthan in solution as influenced by temperature and salt addition. Journal of Molecular Liquids, 287, 111008.

https://doi.org/10.1016/j.molliq.2019.111008

Bueno, V.B., Bentini, R., Catalani, L.H., \& Petri, D.F.S. (2013). Synthesis and swelling behavior of xanthanbased hydrogels. Carbohydrate Polymers, 92(2), 10911099. https://doi.org/10.1016/j.carbpol.2012.10.062

Céline, F., Comesse, S., Renou, F., \& Grisel, M. (2018). Hydrophobically modified xanthan: Thickening and surface active agent for highly stable oil in water emulsions. Carbohydrate Polymers, 205, 362-370. https://doi.org/10.1016/j.carbpol.2018.10.052

Ceulemans, J., Vinckier, I., \& Ludwig, A. (2002). The Use of Xanthan Gum in An Ophthalmic Liquid Dosage Form: Rheological Characterization of the Interaction With Mucin. Journal of Pharmaceutical Sciences, 91(4), 1117-1127. https://doi.org/10.1002/jps.10106

Dário, A.F., Hortêncio, L.M.A., Sierakowski, M.R., Neto, J.C.Q., \& Petri, D.F.S. (2011). The effect of calcium salts on the viscosity and adsorption behavior of xanthan. Carbohydrate Polymers, 84(1), 669-676. https://doi.org/10.1016/j.carbpol.2010.12.047

Hajikhani, M., Khanghahi, M.M., Shahrousvand, M., Mohammadi-Rovshandeh, J., Babaei, A., \& Khademi, S.M.H. (2019). Intelligent superabsorbents based on a xanthan gum/poly (acrylic acid) semi-interpenetrating polymer network for application in drug delivery systems. International Journal of Biological Macromolecules, 139, 509-520.

https://doi.org/10.1016/j.ijbiomac.2019.07.221
Hamcerencu, M., Popa, M., Riess, G., \& Desbrieres, J. (2019). Chemically modified xanthan and gellan to prepare biomaterials for ophthalmic applications. Polymer International, 69(11), 1051-1057. https://doi. org/10.1002/pi.5927

Krstonošić, V., Dokic, L., Dokic, P., \& Dapčević, T. (2009). Effects of xanthan gum on physicochemical properties and stability of corn oil-in-water emulsions stabilized by polyoxyethylene (20) sorbitan monooleate. Food Hydrocolloids, 23(8), 2212-2218. https://doi.org/10.1016/j.foodhyd.2009.05.003

Kulkarni, R.V., Inamdar, S.Z., Das, K.K., \& Biradar, M.S. (2019). 7 - Polysaccharide-based stimuli-sensitive graft copolymers for drug delivery. In S. Maiti \& S. Jana (Eds.), Polysaccharide Carriers for Drug Delivery (pp.155-177). Woodhead Publishing. https://doi. org/10.1016/B978-0-08-102553-6.00007-6

Lallemand, F., Felt-Baeyens, O., Besseghir, K., Behar-Cohen, F., \& Gurny, R. (2003). Cyclosporine A delivery to the eye: A pharmaceutical challenge. European Journal of Pharmaceutics and Biopharmaceutics, 56(3), 307-318. https://doi.org/10.1016/S0939-6411(03)00138-3

Mann, A., Campbell, D., \& Tighe, B.J. (2016). 2 - The ageing ocular surface: Challenges for biomaterials design and function. In T.V. Chirila \& D.G. Harkin (Eds.), Biomaterials and Regenerative Medicine in Ophthalmology (2 ${ }^{\text {nd }}$ Edition, pp.17-43). Woodhead Publishing. https://doi.org/10.1016/B978-0-08100147-9.00002-X

Morsi, N., Ibrahim, M., Refai, H., \& El Sorogy, H. (2017). Nanoemulsion-based electrolyte triggered in situ gel for ocular delivery of acetazolamide. European Journal of Pharmaceutical Sciences, 104, 302-314.

https://doi.org/10.1016/j.ejps.2017.04.013

Rosalam, S., \& England, R. (2006). Review of xanthan gum production from unmodified starches by Xanthomonas comprestris sp. Enzyme and Microbial Technology, 39(2), 197-207.

https://doi.org/10.1016/j.enzmictec.2005.10.019

Sharma, S., \& Batra, S. (2019). Recent advances of chitosan composites in artificial skin: the next era for potential biomedical application. In A.-M. Holban \& A.M. Grumezescu (Eds.), Materials for Biomedical Engineering (pp.97-119): Elsevier. https://doi. org/10.1016/B978-0-12-816909-4.00005-1

Sharma, S., Sinha, V., Sarwal, A., \& Shukla, R. (2018). Chitosan-Based Nanocarriers: A Promising Delivery System for Bioactives. NanoAgroceuticals \& NanoPhytoChemicals (pp. 265-276). CRC Press. https://doi.org/10.1201/9781351139281-13

Benita, S., \& Nassar, T. (2016). US20190209466A1. 
Su, L., Ji, W.K., Lan, W.Z., \& Dong, X.Q. (2003). Chemical modification of xanthan gum to increase dissolution rate. Carbohydrate Polymers, 53(4), 497-499. https://doi.org/10.1016/S0144-8617(02)00287-4

Sumit, S., Shikha, L., \& Rayasa, M. (2012). Potential of chitosan for nose to brain drug delivery. International
Journal of Pharmaceutical Sciences Review and Research, 16(1), 47-55.

Zatz, J.L., \& Knapp, S. (1984). Viscosity of Xanthan Gum Solutions at Low Shear Rates. Journal of Pharmaceutical Sciences, 73(4), 468-471.

https://doi.org/10.1002/jps.2600730410
旬

\section{CHITKARA}

\section{Journal of Pharmaceutical Technology, Research and Management}

Chitkara University, Saraswati Kendra, SCO 160-161, Sector 9-C, Chandigarh, 160009, India

Volume 8, Issue 1

May 2020

ISSN 2321-2217

Copyright: [@2020 Shiveena Bhatia et al.] This is an Open Access article published in Journal of Pharmaceutical Technology, Research and Management (J. Pharm. Tech. Res. Management) by Chitkara University Publications. It is published with a Creative Commons Attribution- CC-BY 4.0 International License. This license permits unrestricted use, distribution, and reproduction in any medium, provided the original author and source are credited. 